This item was submitted to Loughborough's Research Repository by the author.

Items in Figshare are protected by copyright, with all rights reserved, unless otherwise indicated.

\title{
A tunable three-dimensional printed microfluidic resistive pulse sensor for the characterization of algae and microplastics
}

PLEASE CITE THE PUBLISHED VERSION

https://doi.org/10.1021/acssensors.0c00987

\section{PUBLISHER}

American Chemical Society (ACS)

VERSION

AM (Accepted Manuscript)

\section{PUBLISHER STATEMENT}

This document is the Accepted Manuscript version of a Published Work that appeared in final form in ACS Sensors, copyright $($ ) American Chemical Society after peer review and technical editing by the publisher. To access the final edited and published work see https://pubs.acs.org/doi/10.1021/acssensors.0c00987.

\section{LICENCE}

CC BY-NC-ND 4.0

\section{REPOSITORY RECORD}

Pollard, Marcus, Eugenie Hunsicker, and Mark Platt. 2020. "A Tunable Three-dimensional Printed Microfluidic Resistive Pulse Sensor for the Characterization of Algae and Microplastics”. Loughborough University. https://hdl.handle.net/2134/12691760.v1. 
1 A Tunable 3D Printed Microfluidic Resistive Pulse Sensor for the Characterisation of Algae and Microplastics.

3

4

M. Pollard, ${ }^{1}$ E. Hunsicker, ${ }^{1}$ M. Platt ${ }^{1 *}$

5

1 - School of Science, Loughborough University. Epinal Way. LE11 3TU

6

*Corresponding author m.platt@lboro.ac.uk

7

8

9

10

11

12

13

14

15

16

17

18

19

20

21

22

23

24

25

26

27 


\section{Abstract}

29 Technologies that can detect and characterise particulates in liquids have applications 30 in health, food and environmental monitoring. Simply counting the numbers of cells or 31 particles however is not sufficient for most applications, and other physical properties 32 must also be measured. Typically, it is necessary to compromise between chemical 33 and biological specificity of the sensor and its speed. Here we present a low-cost and 34 high-throughput multiuse counter that classifies a particle's size, concentration, and shape. We also report how the porosity/ conductivity or the particle can influence the signal. Using an additive manufacturing process, we have assembled a reusable flow resistive pulse sensor capable of being be tuned in real time to measure particles from $2-30 \mu \mathrm{m}$, across a range of salt concentrations i.e. $2.5 \times 10^{-4}$ to $0.1 \mathrm{M}$. The device remains stable for several days with repeat measurements. We demonstrate its use for characterising algae with spherical and rod structures as well as microplastics shed from teabags. We present a methodology that results in a specific signal for microplastics, namely a conductive pulse, in contrast to particles with smooth surfaces such as calibration particles or algae, allowing the presence of microplastics to be easily confirmed and quantified. In addition, the shape of the signal and particle are correlated, giving an extra physical property to characterise suspended particulates. The technology can rapidly screen volumes of liquid, $1 \mathrm{~mL} / \mathrm{min}$, for the presence of microplastics and algae.

Keywords: Resistive Pulse Sensor, Micro plastic, Algae, Environmental, Food. 
63 Monitoring the presence of particles, be they biological or manmade, within the environment requires a multidisciplinary approach. Microplastics are an increasing cause of concern due to their ubiquity, and their impact within the food chain and upon health is poorly understood. Algae communities are a good indicator species that are sensitive to changes in their habitat. The total biomass of algae and their diversity can be used as indicators of water quality, which makes them a universal indicator species, including the impact on microplastics. ${ }^{1-3} \mathrm{New}$ analytical technologies can aid the characterisation of algae quickly regardless of solution properties, salt levels and turbidity. Whilst current methods can monitor the presence of algae remotely by buoy in-takes, ${ }^{4-6}$ or via satellite imaging to track algal blooms, ${ }^{7}$ these methods are unable to differentiate and quantify biological and manmade particulates.

Resistive Pulse Sensors (RPS), based upon the Coulter Counter principle have seen a resurgence in the last two decades. When integrated into microfluidic systems, this 1950's technology is finding new applications. ${ }^{8-12}$ New manufacturing processes, coupled with improved electronics have enabled them to characterise analytes' sizes $^{13-15}$, concentrations ${ }^{16,17}$, shapes ${ }^{18}$ and charges. ${ }^{19,20}$ As a result, RPS has found numerous applications within environmental monitoring of, for example: bacteria ${ }^{21}$, algae ${ }^{22}$, and heavy metal ions ${ }^{23}$. The sensing system is simple, monitoring the temporary changes in current caused by the translocation of an analyte through a narrow constriction, termed a sensing region. Their limited number of components make them easy to manufacture, requiring only two electrodes, a constriction and a solution containing an electrolyte in order for the sensor to operate. The key aspects which determine the sensitivity in these devices are the sensing regions dimensions, as the signal generated by the analyte is proportional to the volume of the sensing region it excludes. A smaller sensing region can detect smaller analytes, however creating the smaller sensing regions requires advanced manufacturing processes, and they can lead to blockages when larger analytes or aggregates are present.

Additive manufacturing (AM), or 3D printing, has recently gained considerable attention as an alternative to traditional lithography. This method of fabrication allows three dimensional devices to be rapidly produced. One of the most popular types of additive manufacturing is stereolithography (SLA). ${ }^{24-26}$ Here the device is produced layer by layer by exposing a photosensitive polymer to UV light, causing it to cure before a new layer is formed. This produces devices which are water tight with high resolution features using low cost printers ${ }^{27}$. The limitation to the feature size is dependent on the pixel or laser spot size as this determines the minimum curable area. ${ }^{24,26}$ One aspect that stereolithography struggles with is producing microfluidic devices with internal voids or channels. Once these are printed, the resin needs to be flushed out of the void before it is usable. Also over-curing, when the UV light causes resin within the channel to cure, can cause a channel to seal. ${ }^{27}$ Therefore many AM microfluidics have featured channel sizes exceeding $0.5 \mathrm{~mm}^{28,29}$. Recently, Gong et al demonstrated a custom 3D printer which was capable of printing channels with a cross section of $18 \times 20$ microns which is an encouraging sign for AM produced microfluidics. 
Whilst 2-photon polymerisation offers an opportunity to print smaller channels $27,30,31$, the integration of these into higher throughput flow systems has yet to be demonstrated.

We have previously used surface channels within AM devices sealed with a polymer acetate film to overcome the issue of channel blockage, termed gen 1 in this study. Using this method, commercial SLA printers and resins could reproducibility produce channels $\geq 100 \mu \mathrm{m}$, which were used as a gen-1 flow-RPS device. ${ }^{32}$ However, a drawback of this process was the lifetime of the device, as over longer periods e.g. 24 hours, the adhesive on the polymer film weakened, causing it to peel off, or encroach into the channel, blocking it. Here we overcome these issues and extend the sensing dynamic range to smaller particles. The second generation device has been created with a re-sealable easy to change lid and a compressible Polydimethylsiloxane (PDMS) gasket layer that extends the sensing range to detect particles below $5 \mu \mathrm{m}$. The device uses a lid which is held in place via screws, between the base unit and lid sits the PDMS layer. When the screws are tightened, the lid is pushed into the sensing region, reducing the dimensions of the sensing region and increasing the size sensitivity of the device. The lids can be easily interchanged with no modification to the flow device base, meaning in the event of blockage or damage, the lid can be removed the device and cleaned, or the lid replaced with minimal downtime. Here we use the device to characterise size, concentration, and, unique to this study the

\section{Experimental}

128 Chemicals and reagents: CPC2000, 2 micron carboxylated polystyrene calibration particles were obtained from Izon Science Ltd, 10 micron and 20 micron carboxlyated polystyrene calibration particles denoted as CP10M and CP20M were obtained from Izon Science Ltd. 30 Micron carboxylated polystyrene particles, Cat No. 84135, were obtained from Sigma-Aldrich, potassium chloride obtained from Fisher Scientific UK, $>99 \%$ cat no: P/4240/60, Acc Silicones QSil216 was obtained from RS components, catalogue no: 458-765, part no: QSil 216, Algae samples were obtained from Roscoff Culture Collection, Aurantiochytrium Mangrovei (Spherical), catalogue number: RCC893 and Navicula ramosissima (Rod), catalogue number: RCC5374, Isopropanol obtained from VWR.

Data Analysis: Data analysis was performed within the data analysis module of the Izon control suite, baseline extraction was performed using molecular devices clampfit version 10.7, and pulse shape analysis was performed with a custom $\mathrm{R}$ Code.

Device Assembly: In order to assemble the device, the lid was affixed to the base via six machine screws, figure 1, located at each corner, and two screws in the middle of the device. The screws were tightened and secured in place using nuts. Into each one of the screw threads, HPLC fittings were attached in order to accommodate the inlet from the pumps, the electrodes and the outlet. Once fully assembled, the device was 
146 placed into a custom-made faraday cage and electrolyte solution was pumped into the

147 device.

148 Device Printing: Both the lid and base of the device were printed on an Asiga Pico 149 HD27 UV using FORMlabs clear resin. Files were converted from the CAD software, 150 Siemens NX11 to STL and prepared for printing using Asiga Composer software. 151 Once printed, the parts were cleaned and post cured using a UV light box.

152 PDMS Gasket: The PDMS gaskets were formed by mixing parts A and B of QSil 216 153 in a 10:1 ratio. The lid was placed into a petri dish with the ridge oriented to the bottom. 154 The uncured PDMS was poured around the edge of the lid making sure that the whole 155 lid was covered up the ridge and no larger air pockets remained. The PDMS was then 156 cured for one hour at $70^{\circ} \mathrm{C}$ or until set.

157 SEM/EDS and Optical Imaging: Prior to SEM imaging, the samples were sputter 158 coated in Au/Pd for 90 seconds using a Quorum Q150T ES gold sputter coater. SEM 159 images were captured on a Zeiss 1530VP FEGSEM. EDS data was captured using 160 Oxford instruments X-mas $80 \mathrm{~mm}^{2}$ detector and processed using Oxford Instruments 161 Aztec EDS microanalysis software. Microscope images were captured using a Nixon 162 Optiphot 2 optical microscope, and images were captured using a DS 5M camera with 163 a DS-L1 camera control unit.

164 Electrode fabrication: Electrodes were fabricated by inserting a section of silver wire, $1650.25 \mathrm{~mm}$ diameter, 99.99\% purity obtained from Advent Research materials catalogue 166 no: AG5485, into a pipette tip. A small section of the wire is threaded through the narrow end of the pipette, and the wire is glued in place using Araldite $®$ Rapid epoxy resin; the electrodes were then left to dry.

169 Sample run: Samples were loaded into Dolomite mitos p-pump which is controlled via 170 flow control centre software. Once the required pressure has been entered into the 171 software, the pumps then drive the sample into the device. When signals are detected, 172 the recording software is activated and each sample is recorded for the required time 173 or until the required number of particles have been detected. The flow rate was 174 determined by setting the lid to the required baseline and pressure. A pre-weighed 175 Eppendorf was then positioned at the outlet for one minute, then removed and 176 reweighed to determine the mass and volume of the liquid run through the device over 177 that period of time.

178 Tea sample preparation: Tea samples were prepared by making an incision into the 179 bag and discarding the contents. The bags were then washed with deionised water 180 and allowed to dry. Finally, a glass vial of deionised water was heated to $95^{\circ} \mathrm{C}$ and 181 the dried tea bag was placed into the vial for 5 minutes. After 5 minutes, the solution 182 was decanted into another vial and allowed to cool. The sample was then diluted to 183 the required concentration with electrolyte solution. 
184 Tea sample electron microscopy preparation: The samples were prepared by vacuum 185 filtrating the decanted solution using an ANODISK $47 \mathrm{~mm}$ filtration membrane, 0.02 186 micron. The membrane was then washed five times with deionised water, $15 \mathrm{M} \Omega$, and 187 allowed to dry before being mounted onto an aluminium SEM stub using a carbon 188 adhesive tab.

189 Pulse shape analysis: The pulses were analysed by custom R code which has been 190 previously reported ${ }^{18}$. Pulses were extracted, detrended and aligned, then 191 approximated using b-splines (using the COBS package). Logistic models for 192 shape/run discrimination were built on training data using three spline coefficients 193 showing the greatest predictive power for shape classification (under lasso penalised 194 regression using the glmnet package). Classification power was then evaluated on 195 independent testing data.

\section{Results}

197 Figure 1a-d, shows images of the base unit, lid, sensing zone and electrodes. The lids 198 can have several designs; the first is a flat surface that mimics the acetate film from 199 gen 1 . The second design has a ridge that extends $1 \mathrm{~mm}$ out from the surface and is 200 aligned to extend into the channel. The third has multiple ridges that can change the 201 shape of channel and sensing region, figure 1e, and as a particle traverses the sensor 202 characteristic spikes are recorded figure 1f. The device was designed to be integrated 203 into a flow system, thus has printed screw threads to connect pumps. As well as the 204 lid design, a second alteration from gen 1 is the integration of the electrodes' screw 205 threads, see figure 1d. Figure $\mathrm{s} 1$ shows all the dimensions and photographs of the 206 components. 

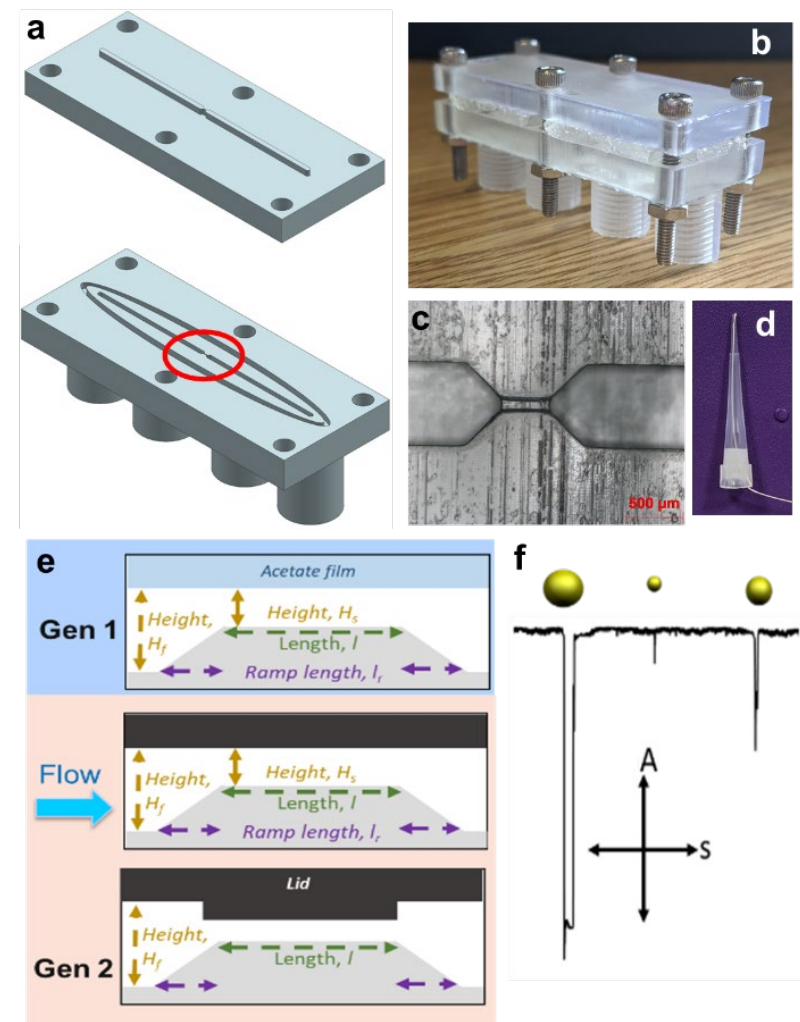

Figure 1 a) CAD design of the device, b) Image of the assembled device, c) an optical microscope image of the sensing zones circled in part a, d) silver wire embedded within a pipette tip, e) schematic of the sensing zone when using an acetate film, a flat lid and ridge lid, f) schematic of the resistive pulses expected when a particle passes through the sensing zone.

214 Optical images of the channel, ridge lid and PDMS layer are shown in figure 2a. The 215 PDMS layer acts as a gasket sealing the components and preventing any leakage. It 216 is held in place by the screws, and the shape or dimensions (internal volume) of the channel can be controlled via two mechanisms. The first is by altering the shape and 218 structures on the lid. As show in figure 1, the lid can be made with a flat surface, or containing protrusions designed to fit within the main channel in base unit. The second option is to use the PDMS layer, which is compressed into the channel upon the application of a force via the screws. Figure 2 shows the schematic of this process, for the flat lid the PDMS covers its entire under surface and as the screw tension is increased, it forces the flexible PDMS gasket into the channel, causing the channel's internal volume to decrease. For the second lid design, the ridge causes the largest change in channel volume upon tightening of the screws, as opposed to the PDMS layer, which does not extent over the ridge itself. 

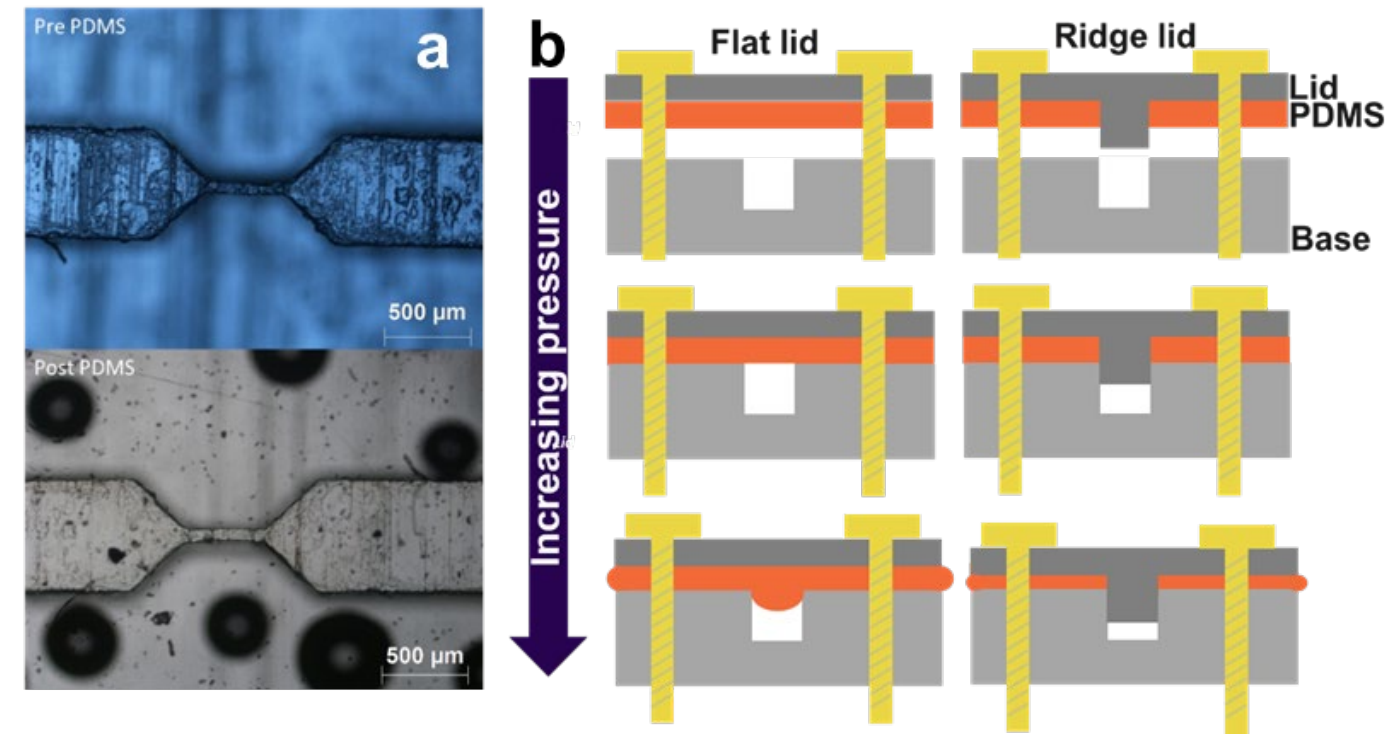

Figure 2a) Optical images of the ridge lid before and after a PDMS coating. b) Schematic of the sealed device with two lid designs.

When the channel is filled with a conductive liquid and a potential difference is applied between the two electrodes, the size of the channel can be monitored in real time through the base line current, $I$, as $I$ is proportional to the sensing volume using the equation 1 the resistance can then be related to the current via Ohm's law ${ }^{33}$ :

$$
\mathrm{R}=\mathrm{L} / \sigma \mathrm{hW},
$$

where $R$ is the resistance, $L$ is the channel length, $\sigma$ is conductivity of solution, and $h$ and $w$ are the height and width of the channel, respectively. To illustrate the stability and response of the device prior to samples we set it up under standard assay conditions. Figure $3 a$ shows the flow rate versus baseline current for the flat lid device under a constant applied pump pressure. By keeping the voltage and ionic strength the same, the total current measured at the baseline can be varied by changing the pressure on the lid through the screws. A higher force, compressed the PDMS layer or ridge further into the channel and resulted in a smaller base line current. A smaller current implied a smaller channel dimension and results in a lower flow rate. The data in figure 3a illustrates the use of a compressible PDMS layer to tune the channel dimensions. Figure $\mathrm{s} 2$ shows the measured flow rate versus applied pump pressure for a variety of channel dimensions. By adding a ridge on the lid that extends into the flow, the shape of the sensing region as well as its internal volume is reduced further, figure S2b. The screws and PDMS compression layer allow further tuning of the 253 channel volume. An extreme version of this allows the ridge to fully seal the channel and acts like a 3D printed valve illustrated elsewhere ${ }^{34,35}$, data not shown. 
a)

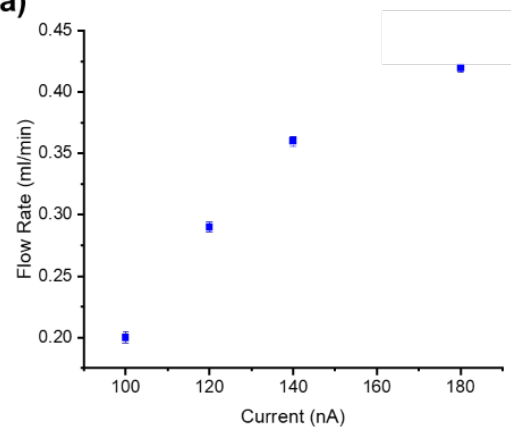

b)

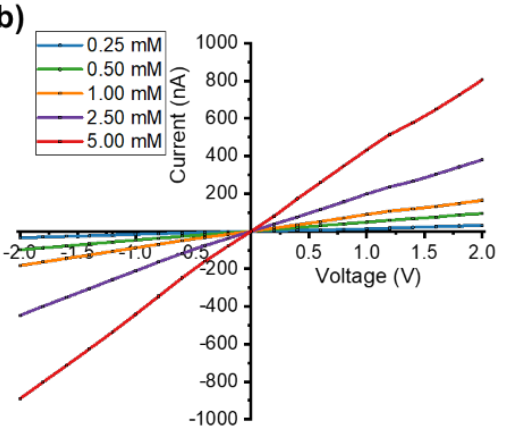

c)

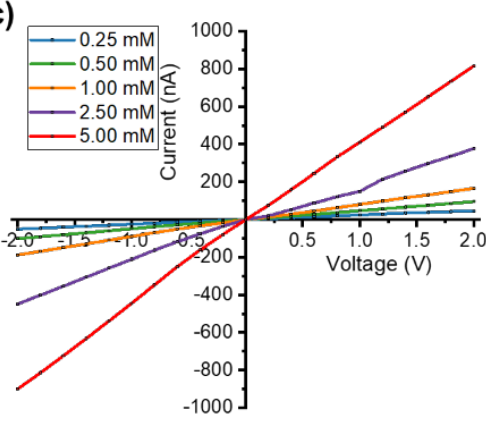

d)

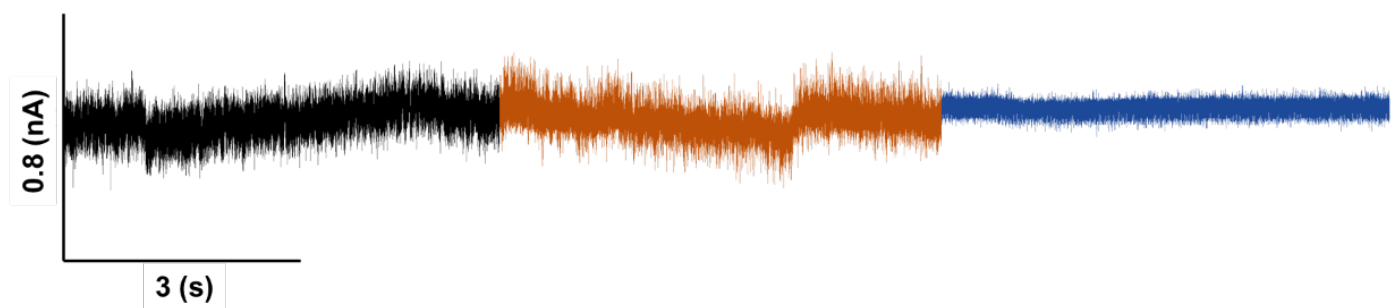

Figure 3. a) Plot of measured current versus flow rate for a flat PDMS lid, voltage $=5.64 \mathrm{~V}, 0.25 \mathrm{mM}$ $\mathrm{KCl}$. Current-Voltage plots for b) Absence of flow c) 100 mbar of pressure applied to the liquid, d) Baseline currents for the device with 100 mbar flow pressure in 100,50 and $0.25 \mathrm{mM} \mathrm{KCl}$ (left to right), Voltage $=0.5 \mathrm{~V}(100 \mathrm{mM}), 0.6 \mathrm{~V}(50 \mathrm{mM}), 8.5 \mathrm{~V}(0.25 \mathrm{mM})$, the total current measured under each condition was 810,570 and $120 \mathrm{nA}$.

261 Figure 3 also illustrates the relationship between applied voltage and current for the ridge lid in the presence and absence of convection. The linear relationship between current-voltage and the consistency of the current in the presence of fluid flow, shows no preferential current flow/rectification or problems with resistance between the electrodes. To test any effect that the lid material may have on the current, the base unit was sealed with an acetate film to mimic gen-1, figure s2. Also shown in figure $3 d$ is example baseline current traces and noise during convection for various ionic strengths. It should be noted that the base line current density also decreases at lower ionic strengths, and whilst the noise levels decrease from left to right, the proportion of noise to the total current remains comparable.

271 After the channel was sealed with the PDMS gasket and lid, the device was used as a resistive pulse sensor. $20 \mu \mathrm{m}$ particles were added to a sample reservoir and pushed through the device using a range of flow rates. Figure $4 a$ shows example current vs time trace, and the relationship between pulse frequency and flow rate, figure $4 \mathrm{~b}$. The pulse frequency, $J$, has linear relationships with flow rate, $\Delta P$, and with concentration, $276 \mathrm{C}_{\mathrm{s}}$, (figure $4 \mathrm{~b}, \mathrm{c}$ ), as predicted by equation 2 , where the contribution from electrophoresis and electroosmosis is negligible given the high flow rates. ${ }^{36}$

279 The blockade magnitudes and durations for figure $4 \mathrm{~b}$ are shown in figure $\mathrm{s} 3$, 280 illustrating that the flow rate does not change the magnitude of the signal, but does 
a)

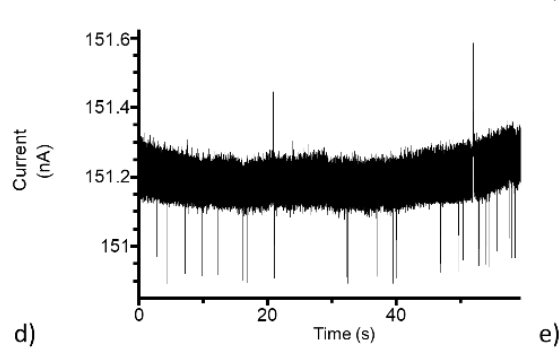

d)

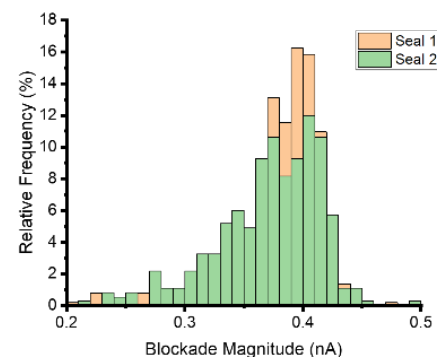

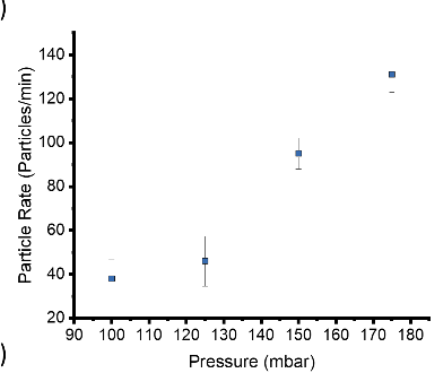

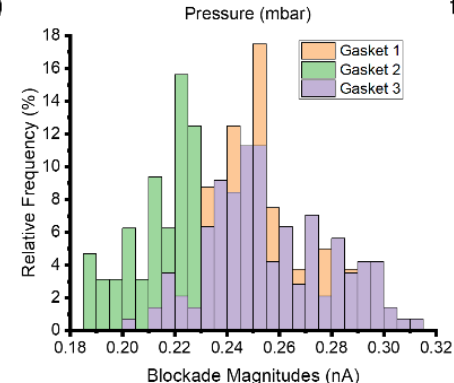

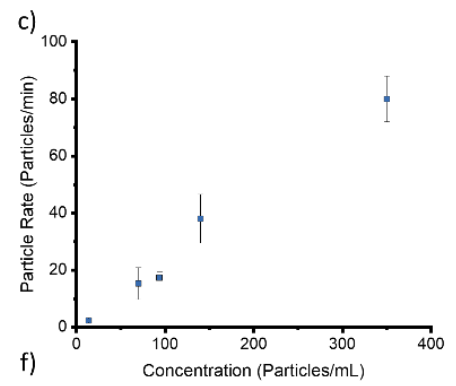

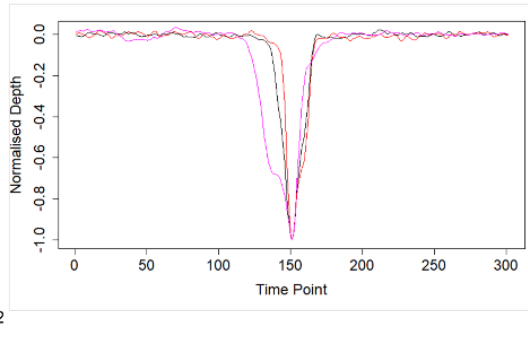

Figure 4 All examples were run on $0.25 \mathrm{mM} \mathrm{KCl}$ a) Example current trace from a ridge lid design, particles $20 \times 10^{-6} \mathrm{~m}$ in diameter, $8.5 \mathrm{~V}$. b) Particle count versus flow rate, particles $20 \times 10^{-6} \mathrm{~m}$ in diameter, $8.5 \mathrm{~V}$. c) Particle count versus concentration, particles $20 \times 10^{-6} \mathrm{~m}$ in diameter, $8.5 \mathrm{~V}$. d) Distribution of pulse magnitudes, particles $30 \times 10^{-6} \mathrm{~m}$ in diameter, $5.64 \mathrm{~V}$, before and after the same device and PDMS gasket was reassembled. e) Distribution of pulse magnitudes, particles $20 \times 10^{-6} \mathrm{~m}$ in diameter, voltage $=7.5 \mathrm{~V}$, using three different PDMS gaskets. f) Average pulse shape for each gasket in e, average of over $>60$ particles

The same PDMS gasket could be reused three times before any leakages were observed, each time the components were placed together and the tension on the screws could be adjusted to match the baseline current. This was interpreted as producing a channel of similar dimensions. Example data sets from the same device and PDMS gasket taken apart and resealed are show in figure $4 \mathrm{~d}$. The device could remain sealed for four days without any deterioration in signal quality, figure $\mathrm{s} 4$, although it should be noted that on each day a calibration is needed to be run to quantify the analyte as there is some drift in the signal. To illustrate the reproducibility of different PDMS gaskets, the same device was sealed with three different PDMS layers. Figure $4 \mathrm{e}$ which shows the blockage distributions of $20 \mu \mathrm{m}$ particles using the same base and lid unit with the different PDMS gaskets, with the average pulse shape from hundreds of translocations in each setup shown in figure $4 \mathrm{f}$. The reproducible pulse height demonstrates the consistency of the channel dimensions.

It is important to note that here the shape of the pulse reflects the internal shape of the sensing zone. Whilst we hypothesised that the signal should be a rectangular pulse with a flat and consistent current during the translation event, the pulse shape indicates a different relationship. Similar effects were seen in the previous examples, and future work is needed to model the current and behaviour of the signal. We also 
310 to match the base line current a consistent sensing zone could be produced each time.

311 The pulse shape can be dependent upon the flow rate, as this effects the time within 312 the channel, figure $\mathrm{s} 5$ shows the flow average pulse shape for spherical particles 313 across a range of flow rates.

314 Changing the volume of the sensing zones allows the sensitivity of the RPS to be 315 tuned. An example of this is shown in figure 5a. The same base, lid unit and PDMS 316 gasket were assembled. Upon tightening the screws, the blockage magnitude of the 317 same sized particles increases. The result matches the theory for RPS sensors. 318 Where the same sized particle blocks a larger proportion of the channel, resulting in 319 larger blockade current. With this ability the same device, printed with an initial channel 320 dimensions of $100 \times 100 \times 500 \mu \mathrm{m}$ could measure particles from 2 to $30 \mu \mathrm{m}$ figure $5 \mathrm{~b}-\mathrm{e}$. 321 This simple configuration enables an enhanced dynamic range on a single set of 322 reusable components.

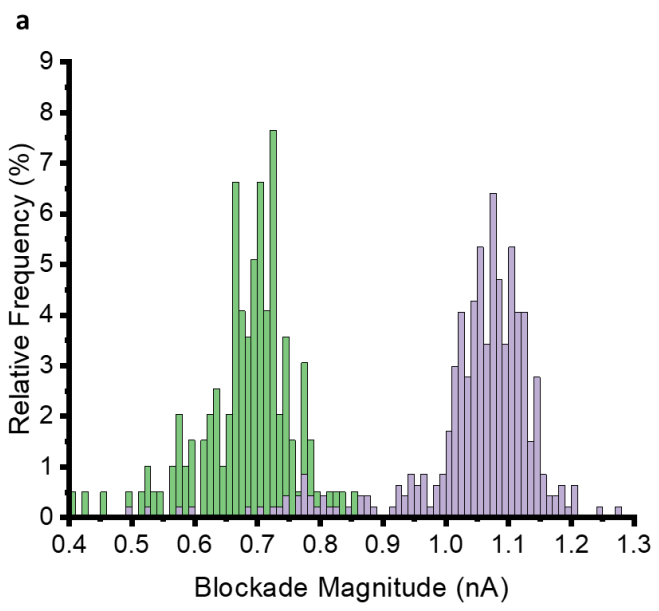

323

324

325

326

327

328

329
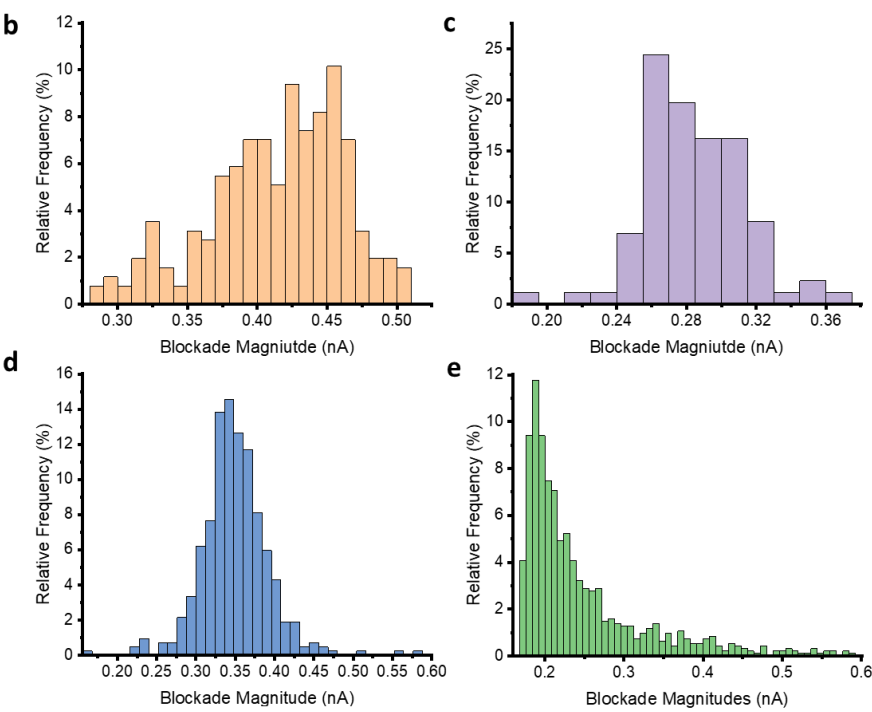

Figure 5 a) Blockade distributions for $30 \times 10^{-6} \mathrm{~m}$ in diameter particles Voltage $=5.64 \mathrm{~V}, 0.25 \mathrm{mM} \mathrm{KCl}$, using two different (low - green, high - purple) screw tensions. b) As a control, acetate tape (Gen-1) was used to seal the device. $30 \times 10^{-6} \mathrm{~m}$ in diameter particles Voltage $=5.64 \mathrm{~V}, 0.25 \mathrm{mM} \mathrm{KCl}$. Increasing the tension on the screws allows the RPS to measure smaller particles. c) $20 \times 10^{-6} \mathrm{~m}$ in diameter particles Voltage $=8.5 \mathrm{~V}, 0.25 \mathrm{mM} \mathrm{KCl}$, d) $10 \times 10^{-6} \mathrm{~m}$ in diameter particles Voltage $=0.6 \mathrm{~V}, 100 \mathrm{mM}$ $\mathrm{KCl}$. e) $2 \times 10^{-6} \mathrm{~m}$ in diameter particles Voltage $=1 \mathrm{~V}, 100 \mathrm{mM} \mathrm{KCl}$.

The device was designed to screen environmental and food samples for contamination. An emerging threat to the global environment is that of microplastics, thus a first test was inspired by a recent publication. Hernandez and co-workers found that certain tea bags shed billions of plastic microparticles during their use. Follow their published protocol ${ }^{37}$, we placed the bags into hot water for 5 mins, and passed the solution through our sensor. Within seconds, particles could be observed translocating the device, figure $6 \mathrm{a}$. Using a particle of known size and concentration as a alibrant we calculated the average size to be $21.9 \mu \mathrm{m}$ at $6.52 \times 10^{3}$ particles $/ \mathrm{mL}$. We acknowledge that unlike in the previous study we cannot be counting the entire particle size range and the number of nanoplastics are not included in this value. Teabags from several 
340 manufacturers were tested, and the total number of particles shed from one teabag 341 was as high as $6.52 \times 10^{4}$, table s1. The presence of the particles was consistent 342 across several tea manufactures, see figure s5, and analysis via SEM confirmed the 343 particles were carbon based, figure s5.

344 Varying the ionic strength of the liquid produced interesting effects, figure 6b. At lower 345 ionic strengths, the pulse direction inverted and conductive pulses are prominently 346 recorded. The inversion of the pulse could be caused by two factors, the first is that 347 the polymers may contain a higher concentration of ions, making them "conductive" 348 relative to the surrounding liquid; similar effects have been seen in hydrogels. ${ }^{38}$ 349 Alternatively, the high surface area seen in the SEM figure s5, combined with the lower 350 ionic strength and a larger double layer, could result in a dense ion cloud around each 351 particle that increase the conductivity of the liquid during each translocation. When the 352 same polymer purchased with a smooth surface (figure $s 5 b$ ), was placed into the 353 device, only resistive pulses were recorded even at low ionic strengths. Porous silica 354 particles (figure $\mathrm{s} 5 \mathrm{c}$ ) found within toothpastes also produced predominantly 355 conductive pulses at lower ionic strengths, table s2. Whilst the conductive pulse gives 356 an indication of a change in physical properties, the lack or suitable calibrants makes 357 it harder to size the particles using conductive pulses. For a fully automated screening 358 device, confirmation of the chemical nature of the particles may require additional 359 sensors embedded into the channels and is the scope of future work. However, the 360 pulse direction may give an early indication of manmade particles.

361 Within oceans, counting plastics in the presence of biological particles is challenging. 362 The results in figure 6, however, suggest that bacteria and algae particles, which have 363 fairly smooth surfaces, should also give resistive pulses. This is confirmed within the 364 data shown in Figure $6 \mathrm{c}$ and d. Here a series of data collected for algae particles 365 across a range of ionic strengths is presented, and optical images for the algae are 366 shown in figure s9. Figure 6 demonstrates that the algae always produce resistive 367 pulses across all ionic strengths. This interesting observation may allow scientist to 368 rapidly screen samples for the presence of plastics. 


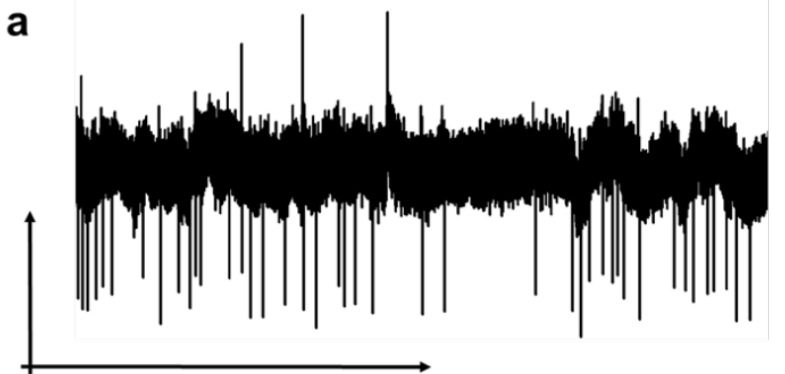

C

b

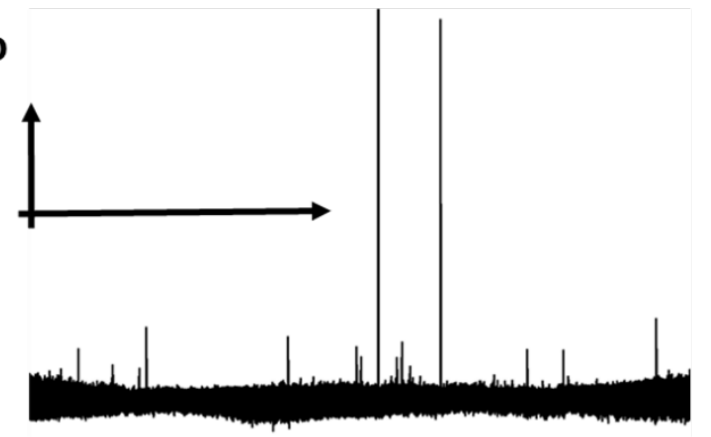

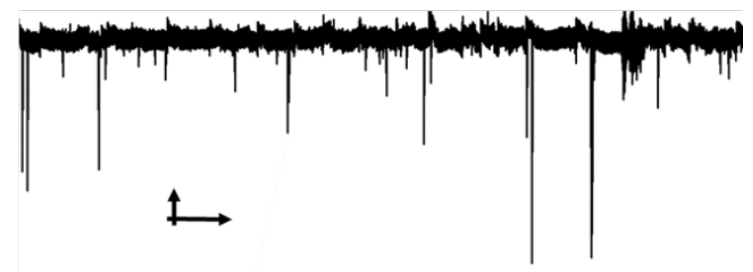

d

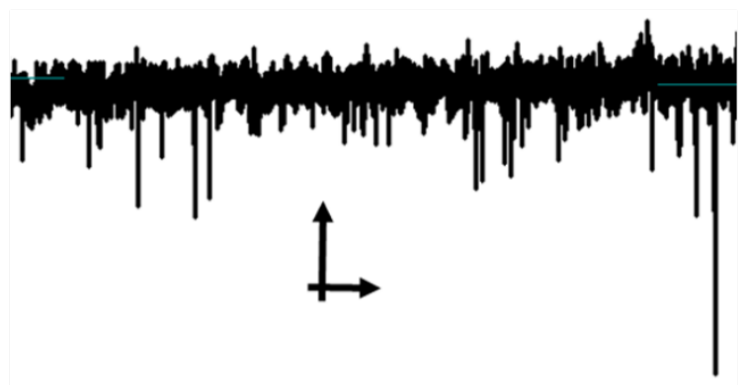

373

Figure 6 a) Microplastic particles run in $50 \mathrm{mM} \mathrm{KCl}$, voltage, $0.4 \mathrm{~V}$, b) Microplastic particles run in 0.25 $\mathrm{mM} \mathrm{KCl,} 7.5 \mathrm{~V} \mathrm{c}$ ) Algae particles run in $50 \mathrm{mM} \mathrm{KCl,} 0.7 \mathrm{~V}$, and d) $0.25 \mathrm{mM} \mathrm{KCl} 7 \mathrm{~V}$. In all images scale bar is $x=10$ sec. $y=0.5 n A$.

Diatoms and algae are known to come in a variety of shapes depending upon the strain. Thus, a description of particle shape may help with the identification. Here we placed some rod-shaped algae (Navicula ramosissima) and some spherical algae (Aurantiochytrium Mangrovei) through the device. We have previously shown how the shape of a particle can be inferred from the shape of the pulse it generates. ${ }^{18}$ Figure 7 shows the average depth-normalised pulse shapes for the two algae strains and their corresponding calibration bead runs. In order to quantify the differences between the pulses arising from algae and calibrants, the pulses were approximated by splines and the spline coefficients compared using a modelling method previously reported. ${ }^{18}$ For these models the splines 5 (just before pulse minimum), 10 and 11 (around the second peak in each pulse) were used, as they showed the greatest differences between the algae and calibrants. See figure S10 for each of the splines. For each type of algae, the model and device were calibrated against a sphere run under the same assembly. When comparing the calibrants to the algae, the model was able to correctly identify $87 \%$ of algae spheres and $86 \%$ of algae rods. This signifies that most of the algae can be identified against a spherical calibrant, see figure S11 for ROC graphs and result table S3. For comparison, when two runs of calibration spheres were run under the same assembly, the model correctly identified the run for only $66 \%$ of the spheres, which is close to the random $50 \%$ correct classification expected when pulses from two runs are identical. A surprising outcome of the data sets was the ability to identify spherical algae. Whilst their general shape should be similar to the calibrants, the algae have a flexible surface which may be deformed within the fluidic system. The deformation of biological particles has been explored for cells and 
exosomes, $17,38,39$ and here the same feature may help differentiate the biological particle from manmade ones. This establishes that the device can be used for shape analysis, and this is likely to improve with an optimised run method and model. A second unexpected and favourable outcome from the analysis was that the device was capable of shape analysis across multiple uses, e.g. days and assemblies, as long as it was calibrated each time; this is in contrast to the current commercial system that the model was originally developed for, which lost the ability to detect shape permanently after a much shorter period of use. The flow rate used to count the algae also affects the quality of the pulse shape information; as shown in figure $7 \mathrm{~b}$, the shape of the pulses from the two strains converge at higher flow rates. Whilst higher sampling frequencies may overcome this in future designs, this was beyond the scope of the current work.

a)

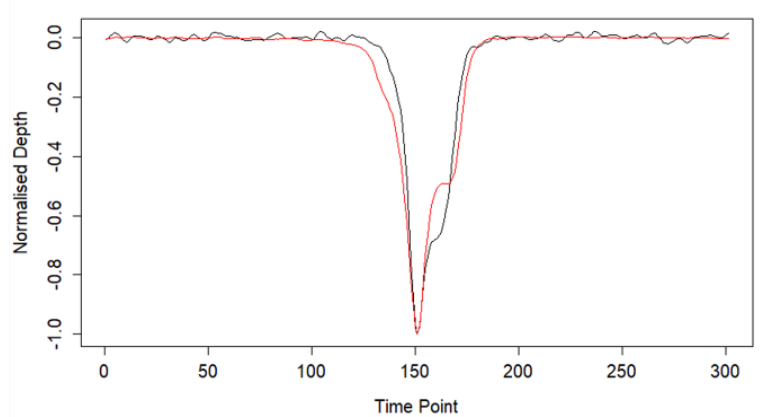

b)

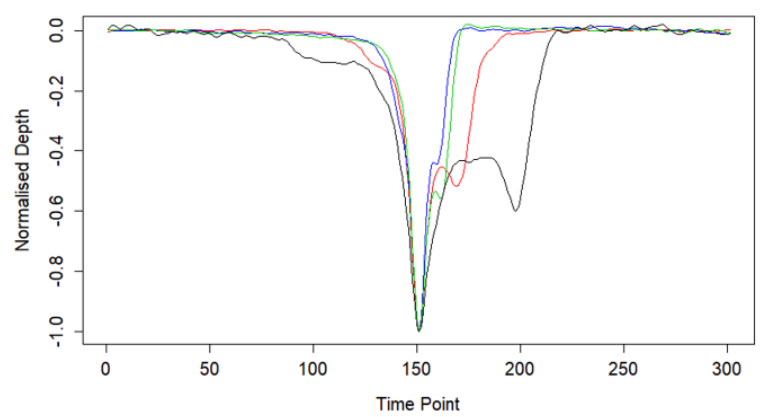
412 Figure 7 a) average pulse shapes of the spherical algae (black) to the rod shape algae (red), b) The
413 average pulse shape for the rod algae across pressures, 50 mbar (black), 100 mbar (red), $125 \mathrm{mbar}$ 414 (green) and 150 mbar (blue).

415 In summary we have demonstrated a tuneable microfluidic RPS device with a reusable 416 lid and base that allows the dimensions of the pore to be tuned in real time to the 417 dimensions of the analyte. The device was able to detect particles as small as 2 418 microns in diameter; representing a ten-fold improvement in sensitivity on the previous 419 generation of the device, which was limited by the resolution of the 3D printer. The 420 device has been fully characterised including the effects of concentration, pressure 421 and reassembling the device. The lowest concentration detected by the device was 42214 particles per $\mathrm{mL}$ at a pressure of $100 \mathrm{mbar}$. The device was then tested with real 423 world cases including the detection of microplastics in tea, where it was discovered 424 that the plastics response to changing electrolyte concentration can be indicative of 425 the material's porosity. The average size and concentration from one manufacture of 426 teabag was determined to be $21.9 \mu \mathrm{m}$ and $6.52 \times 10^{4}$ particles per $\mathrm{mL}$. The device was 427 also able to differentiate between two different shapes of algae in comparison to a 428 calibration sphere, demonstrating that the device can discriminate an analyte on the 429 basis of shape. The current system in a bench top unit operating with a single 430 computer. With the development of small portable current amplifiers and automated 431 software we believe this could be easily deployed in a case for field work and research 432 is underway to facilitate this. 
Acknowledgements:

434 The authors are grateful to the EPSRC Centre for Doctoral Training in Embedded 435 Intelligence under grant reference EP/L014998/1 for financial support.

\section{Supporting Information Available}

437 Supporting Information Available: The following files are available free of charge. 438 Illustration of the design of the model, electric potential and field lines. Images of the 439 RPS created with varying channel sizes, example pulses and signal versus flow rate 440 for multiple devices. This material is available free of charge via the internet at 441 http://pubs.acs.org

\section{References}

444

(1) Liu, G.; Jiang, R.; You, J.; Muir, D. C. G.; Zeng, E. Y. Microplastic Impacts on Microalgae Growth: Effects of Size and Humic Acid. Environ. Sci. Technol. 2020, 54 (3), 1782-1789.

447

(2) Prata, J. C.; da Costa, J. P.; Lopes, I.; Duarte, A. C.; Rocha-Santos, T. Effects of Microplastics on Microalgae Populations: A Critical Review. Sci. Total Environ. 2019, 665, 400-405.

(3) Wu, Y.; Guo, P.; Zhang, X.; Zhang, Y.; Xie, S.; Deng, J. Effect of Microplastics Exposure on the Photosynthesis System of Freshwater Algae. J. Hazard. Mater. 2019, 374, 219-227.

(4) He, X.; Liu, Y.-L.; Conklin, A.; Westrick, J.; Weavers, L. K.; Dionysiou, D. D.; Lenhart, J. J.; Mouser, P. J.; Szlag, D.; Walker, H. W. Toxic Cyanobacteria and Drinking Water: Impacts, Detection, and Treatment. Harmful Algae 2016, 54, 174-193.

(5) Richardson, T. L.; Lawrenz, E.; Pinckney, J. L.; Guajardo, R. C.; Walker, E. A.; Paerl, H. W.; MacIntyre, H. L. Spectral Fluorometric Characterization of Phytoplankton Community Composition Using the Algae Online Analyser $\AA$. Water Res. 2010, 44 (8), 2461-2472.

(6) McQuaid, N.; Zamyadi, A.; Prévost, M.; Bird, D. F.; Dorner, S. Use of in Vivophycocyanin Fluorescence to Monitor Potential Microcystin-Producing Cyanobacterial Biovolume in a Drinkingwater Source. J. Environ. Monit. 2011, 13 (2), 455-463.

(7) Becker, R. H.; Sultan, M. I.; Boyer, G. L.; Twiss, M. R.; Konopko, E. Mapping Cyanobacterial Blooms in the Great Lakes Using MODIS. J. Great Lakes Res. 2009, 35 (3), 447-453.

(8) Zhang, W.; Hu, Y.; Choi, G.; Liang, S.; Liu, M.; Guan, W. Microfluidic Multiple Cross-Correlated Coulter Counter for Improved Particle Size Analysis. Sensors Actuators B Chem. 2019, 296 (April), 126615. 
(9) Song, Z.; Li, M.; Li, B.; Yan, Y.; Song, Y. Automatic Detecting and Counting Magnetic Beads-Labeled Target Cells from a Suspension in a Microfluidic Chip. Electrophoresis 2019, 40 (6), 897-905.

(10) Zhou, T.; Song, Y.; Yuan, Y.; Li, D. A Novel Microfluidic Resistive Pulse Sensor with Multiple Voltage Input Channels and a Side Sensing Gate for Particle and Cell Detection. Anal. Chim. Acta 2019, 1052, 113-123.

(11) Sun, J.; Kang, Y.; Boczko, E. M.; Jiang, X. A Microfluidic Cell Size/density Sensor by Resistive Pulse Detection. Electroanalysis 2013, 25 (4), 1023-1028.

479

(12) Khodaparastasgarabad, N.; Mohebbi, A.; Falamaki, C. A Novel Microfluidic High-Throughput Resistive Pulse Sensing Device for Cells Analysis. Microsyst. Technol. 2019, 25 (10), 3643-3653.

(13) Bernabini, C.; Holmes, D.; Morgan, H. Micro-Impedance Cytometry for Detection and Analysis of Micron-Sized Particles and Bacteria. Lab Chip 2011, 11 (3), 407-412.

(14) Vogel, R.; Willmott, G.; Kozak, D.; Roberts, G. S.; Anderson, W.; Groenewegen, L.; Glossop, B.; Barnett, A.; Turner, A.; Trau, M. Quantitative Sizing of Nano/Microparticles with a Tunable Elastomeric Pore Sensor. Anal. Chem. 2011, 83 (9), 3499-3506.

(15) Weatherall, E.; Hauer, P.; Vogel, R.; Willmott, G. R. Pulse Size Distributions in Tunable Resistive Pulse Sensing. Anal. Chem. 2016, acs.analchem.6b01818.

491

492

493

494

495

496

497

498

499

500

501

502

503

(16) Grabarek, A. D.; Weinbuch, D.; Jiskoot, W.; Hawe, A. Critical Evaluation of Microfluidic Resistive Pulse Sensing for Quantification and Sizing of Nanometer- and Micrometer-Sized Particles in Biopharmaceutical Products. J. Pharm. Sci. 2019, 108 (1), 563-573.

(17) Hinkle, P.; Westerhof, T. M.; Qiu, Y.; Mallin, D. J.; Wallace, M. L.; Nelson, E. L.; Taborek, P.; Siwy, Z. S. A Hybrid Resistive Pulse-Optical Detection Platform for Microfluidic Experiments. Sci. Rep. 2017, 7 (1), 10173.

(18) Maugi, R.; Hauer, P.; Bowen, J.; Ashman, E.; Hunsicker, E.; Platt, M. A Methodology for Characterising Nanoparticle Size and Shape Using Nanopores. Nanoscale 2020, 12 (1), 262-270.

(19) Blundell, E. L. C. J.; Vogel, R.; Platt, M. Particle-by-Particle Charge Analysis of DNA-Modified Nanoparticles Using Tunable Resistive Pulse Sensing. Langmuir 2016, 32 (4), 1082-1090.

(20) Kozak, D.; Anderson, W.; Trau, M. Tuning Particle Velocity and Measurement Sensitivity by Changing Pore Sensor Dimensions. Chem. Lett. 2012, 41 (10), 1134-1136.

(21) Song, Y.; Zhang, H.; Chon, C. H.; Chen, S.; Pan, X.; Li, D. Counting Bacteria on a Microfluidic Chip. Anal. Chim. Acta 2010, 681 (1-2), 82-86.

(22) Song, Y.; Wang, J.; Yang, J.; Wu, Y.; Li, N.; Gong, N.; Pan, X.; Sun, Y.; Li, D. ALGAE DETECTION AND SHIP'S BALLAST WATER ANALYSIS BY A MICROFLUIDIC LAB-ON-CHIP DEVICE. Instrum. Sci. Technol. 2012, 40 (4), 
(23) Heaton, I.; Platt, M. Peptide Nanocarriers for Detection of Heavy Metal lons Using Resistive Pulse Sensing. Anal. Chem. 2019, 91 (17), 11291-11296.

(24) Gale, B.; Jafek, A.; Lambert, C.; Goenner, B.; Moghimifam, H.; Nze, U.; Kamarapu, S. A Review of Current Methods in Microfluidic Device Fabrication and Future Commercialization Prospects. Inventions 2018, 3 (3), 60.

(25) Capel, A. J.; Edmondson, S.; Christie, S. D. R.; Goodridge, R. D.; Bibb, R. J.; Thurstans, M. Design and Additive Manufacture for Flow Chemistry. Lab Chip 2013, 13 (23), 4583.

(26) Bhattacharjee, N.; Urrios, A.; Kang, S.; Folch, A. The Upcoming 3D-Printing Revolution in Microfluidics. Lab Chip 2016, 16 (10), 1720-1742.

(27) Waheed, S.; Cabot, J. M.; Macdonald, N. P.; Lewis, T.; Guijt, R. M.; Paull, B.; Breadmore, M. C. 3D Printed Microfluidic Devices: Enablers and Barriers. Lab Chip 2016, 16 (11), 1993-2013.

(28) Gong, H.; Bickham, B. P.; Woolley, A. T.; Nordin, G. P. Custom 3D Printer and Resin for $18 \mathrm{Mm} \times 20 \mathrm{Mm}$ Microfluidic Flow Channels. Lab Chip 2017, 17 (17), 2899-2909.

(29) Lee, K. G.; Park, K. J.; Seok, S.; Shin, S.; Kim, D. H.; Park, J. Y.; Heo, Y. S.; Lee, S. J.; Lee, T. J. 3D Printed Modules for Integrated Microfluidic Devices. RSC Adv. 2014, 4 (62), 32876-32880.

(30) Perrucci, F.; Bertana, V.; Marasso, S. L.; Scordo, G.; Ferrero, S.; Pirri, C. F.; Cocuzza, M.; El-Tamer, A.; Hinze, U.; Chichkov, B. N.; et al. Optimization of a Suspended Two Photon Polymerized Microfluidic Filtration System. Microelectron. Eng. 2018, 195, 95-100.

(31) Gross, B. C.; Erkal, J. L.; Lockwood, S. Y.; Chen, C.; Spence, D. M. Evaluation of 3D Printing and Its Potential Impact on Biotechnology and the Chemical Sciences. Anal. Chem. 2014, 86 (7), 3240-3253.

(32) Hampson, S. M.; Pollard, M.; Hauer, P.; Salway, H.; Christie, S. D. R.; Platt, M. Additively Manufactured Flow-Resistive Pulse Sensors. Anal. Chem. 2019.

(33) Godwin, L. A.; Deal, K. S.; Hoepfner, L. D.; Jackson, L. A.; Easley, C. J. Measurement of Microchannel Fluidic Resistance with a Standard Voltage Meter. Anal. Chim. Acta 2013, 758, 101-107.

(34) Gong, H.; Woolley, A. T.; Nordin, G. P. High Density 3D Printed Microfluidic Valves, Pumps, and Multiplexers. Lab Chip 2016, 16 (13), 2450-2458.

(35) Riordon, J.; Mirzaei, M.; Godin, M. Microfluidic Cell Volume Sensor with Tunable Sensitivity. Lab Chip 2012, 12 (17), 3016.

(36) Blundell, E. L. C. J.; Mayne, L. J.; Billinge, E. R.; Platt, M. Emergence of Tunable Resistive Pulse Sensing as a Biosensor. Anal. Methods 2015, 7 (17), 7055-7066. 
560

561

562

563

564

565

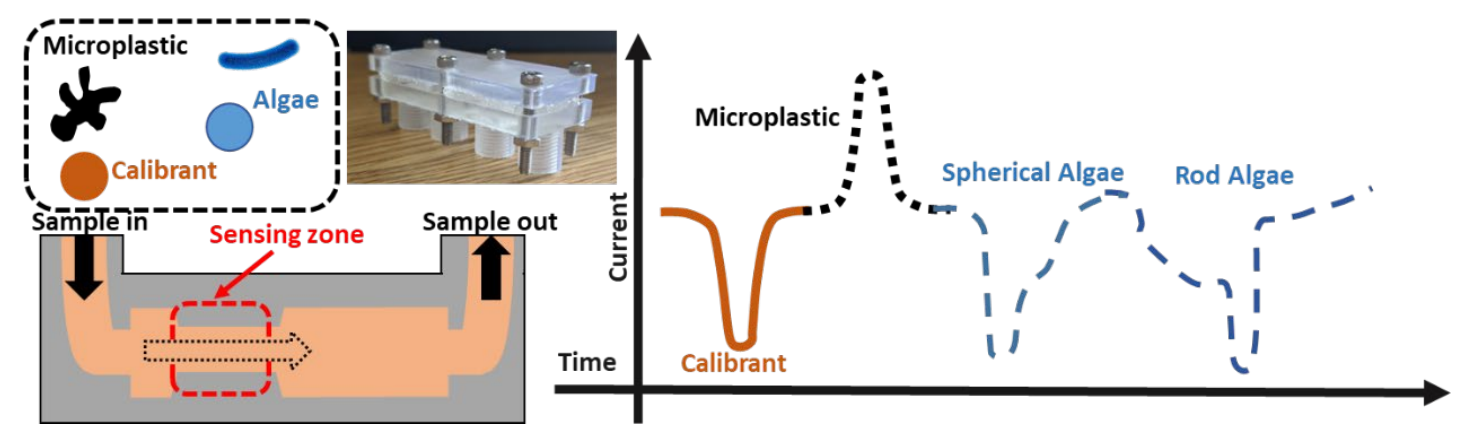

567 TOC image 